determine the specific mutations. A small number of people carry mutations associated with sweat chloride levels in the normal range. If suspicion of CF is high, genotyping should be undertaken in such patients.

There are also a small number of children who have a label of CF following neonatal screening but in whom little or no phenotypic manifestations of CF develop over subsequent years, calling into question the diagnosis (false positives). The second diagnostic algorithm is helpful in such patients as they are likely to have had a CFTR DNA test with only one or no mutations, but may not have had a sweat test since the time of diagnosis. This is not a common occurrence but the algorithm will be helpful in those cases. The sweat test should be repeated and other investigations arranged as appropriate to explore other diagnoses.

In the two algorithms the authors argue that the traditional upper limit for sweat chloride of $40 \mathrm{mmol} / \mathrm{l}$ should be lowered to $30 \mathrm{mmol} / \mathrm{l}$. From their literature review this seems to be a reasonable suggestion, but care in the interpretation of sweat chloride tests in this range will be important in order to avoid false positive diagnoses. Most national guidelines still recommend that levels below $40 \mathrm{mmol} / \mathrm{l}$ are normal. ${ }^{9}{ }^{10}$

The paper also explores the value of adjunctive diagnostic investigations such as nasal PD measurements. These are not widely available and are difficult measurements to make accurately and reproducibly. A number of centres in the UK offer this service and it can be of some value, particularly in confirming that there is no CFTR dysfunction in a patient with an equivocal sweat chloride concentration who has a phenotype suggestive of CF but in whom only one or no mutations can be identified. It is likely that such patients have some other cause for their bronchiectasis. It is unnecessary for every CF centre to have this test available. The performance of nasal PD testing is technically challenging and it is preferable that a few centres develop this expertise. Intestinal current measurements are also valuable but are available at even fewer centres. This involves taking a small biopsy sample from the lower gastrointestinal tract and measuring the electrical current under different conditions. This assessment gives similar information to nasal PD and can confirm or refute dysfunction of the CFTR chloride channel.

The spectrum of the diagnosis of CF and related disorders has widened considerably and should be considered in all patients with bronchiectasis, idiopathic pancreatitis, severe sinusitis, nasal polyps, ABPA and CBAVD, and investigated as appropriate. These algorithms will be a help to clinicians considering or reconsidering the diagnosis.

Thorax 2006;61:556-557.

doi: 10.1136/thx.2005.056309

\section{Authors' affiliations}

J S Elborn, Respiratory Medicine Group,

Queen's University, Belfast, UK

J M Bradley, Health and Rehabilitation

Sciences Research Institute, University of

Ulster, UK
Correspondence to: Professor J S Elborn, Professor of Respiratory Medicine, Queen's University, Belfast and Director of the Northern Ireland Adult Cystic Fibrosis Centre, City Hospital, Belfast BT9 7AB, UK; stuart.elborn@ bch.n-i.nhs.uk

Funding: none.

Competing interests: none declared.

\section{REFERENCES}

Rosenstein BJ, Cutting GR. The diagnosis of cystic fibrosis: a consensus statement. J Pediatr 1998; 132:589-95

2 Karczeski BA, Cutting GR. Diagnosis of cystic fibrosis, CFTR-related disease and screening in cystic fibrosis in the 21 st century. Basel: Karger AG, 2005:2-10.

3 McCloskey M, Redmond AOB, Elborn JS Clinical features associated with delayed diagnosis of cystic fibrosis. Respiration 2000;67:402-8.

4 Nissim-Ratina M, Linde L, Karem B. The CFTR gene: structure, mutations and specific therapeutic approaches. Basel: Karger AG 2005:69-76.

5 Mak V, Zielenski J, Tsui LC, et al. Proportion of cystic fibrosis gene mutations not detected by routine testing in men with obstructive azoospermia. JAMA 1999;281:2217-24.

6 Chillon M, Casals T, Mercier B, et al. Mutations in the cystic fibrosis gene in patients with congenital absence of the vas deferens. N Engl J Med 1995; 332:1475-80.

7 Knowles MR, Durie PR. What is cystic fibrosis? N Engl J Med 2002;347:439-42.

8 De Boeck K, Wilschanki M, Castellani C, et al. Cystic fibrosis: terminology and diagnostic algorithms. Thorax 2006;61:627-35.

9 Association of Clinical Biochemistry. Guidelines for the performance of the sweat test for the investigation of cystic fibrosis in the UK. Report from the Multidisciplinary Working Group, 2002. Available at http:// www.acb.org.uk.

10 National Committee for Clinical Laboratory Standards (NCCLS). Sweat testing: sample collection and quantitative analysis. Approved guideline C34-A2. Wayne, PA: NCCLS, 2000.

\title{
The burden of lung disease
}

\section{R Hubbard}

\section{A timely reminder of the needs of people with respiratory disease in the UK}

$\mathrm{T}$

he range of clinical conditions included under the umbrella of "respiratory medicine" is wide. From cancers to obstructive sleep apnoea, interstitial lung disease to airways disease, occupational lung disease to respiratory infections, there is a variety present in respiratory medicine not seen in other hospital based specialties. This diversity makes respiratory medicine a deeply rewarding specialty in which to work, but also means that it is not easy to quantify the full impact of lung disease on the health of the British public.

For this reason, the British Thoracic Society has produced the second edition of "The Burden of Lung Disease" which includes a number of statistics that may be startling to the casual reader and of interest to those involved in resource allocation in the NHS. ${ }^{1}$ For example, of the 580000 deaths each year in the UK, one in five is due to respiratory disease with 35000 deaths from lung cancer, 34000 from pneumonia, and 27000 from COPD. Respiratory disease now accounts for more than 845000 hospital admissions each year and is second only to injury and poisoning as a cause of emergency admission to hospital. Asthma remains the most common chronic illness in children. The estimated cost to the UK of respiratory disease in 2004 was a staggering £6.6 billion. Clearly, the impact of lung disease is huge.

The report also provides evidence of health inequalities in lung disease. The socioeconomic gradient in death rates from respiratory disease is steeper than that for all cause mortality, highlighting the great potential to prevent deaths from lung disease. Worryingly, the report also suggests that respiratory medicine in the UK is falling behind other specialties and other countries. For example, the death rate from ischaemic heart disease in the UK has 
halved over the last 20 years, but that due to respiratory disease is essentially unchanged and death rates from lung cancer in the UK are among the worst in Europe.

The scale of the problem of lung disease detailed in the report is impressive, but is still likely to be an underestimate because data are just not available for some important respiratory diseases. For example, we know that in some populations obstructive sleep apnoea is common and associated with a range of morbidities, but we still need information on the full public health impact of this condition..$^{2-4}$ We have data from death registrations that the incidence of idiopathic pulmonary fibrosis is rising steeply, but that these data underestimate greatly the true frequency of the disease. ${ }^{5}{ }^{6}$ There is still a considerable amount of research required to allow the third edition of "The Burden of Lung Disease" to describe the impact of the full spectrum of lung disease.

In his foreword John Macfarlane comments that the "report confirms that we urgently need to provide an improved NHS service for those with lung disease". I would echo these thoughts and add that we also need an improved up to date evidence base. The challenge now is how best to make progress.

In the long term, the solution to preventing deaths from lung cancer and COPD lies with drastically reducing the prevalence of smoking in the UK. This would also remove much of the socioeconomic gradient for respiratory mortality and have a beneficial impact on a wide range of other respiratory diseases. ${ }^{7}$ Since respiratory medicine has more to gain by reducing the prevalence of smoking than other areas of medicine, it is vital that our specialty takes a lead in promoting and widening access to smoking cessations services. We should also take a leading role in research to improve the efficacy of smoking cessation treatments and to determine how best to deliver services to all sectors of society.

We also urgently need to improve the care for our patients presenting with lung disease now. In some areas more research is needed, but in others the evidence is already strong and the problem is one of service provision. Examples where a strong evidence base already exists, but where many centres have struggled for resources to provide services, include pulmonary rehabilitation for patents with COPD and continuous positive airways pressure for patients with obstructive sleep apnoea. ${ }^{8}$ These examples highlight the need for a coordinated approach to provide these effective but relatively low cost interventions to all the people who need them wherever they live. An example where additional clinical evidence would have a large clinical impact is that of surgical resection rates for lung cancer. Surgical resection rates for lung cancer in the UK are lower than those in Europe, partly because people in the UK present later with more advanced disease. ${ }^{10}$ At the moment we do not know the most effective way to diagnose cases of lung cancer earlier, but possible approaches include publicity/education campaigns and screening programmes, perhaps using low dose spiral CT scanning. ${ }^{11}$ The challenge to the research community it to determine which is the most cost effective approach. With this in mind, lung cancer needs more than the $4 \%$ of the UK cancer research budget that it currently receives. The equivalent figures for breast cancer, colon cancer and ovarian cancer are $17 \%, 11 \%$ and $6 \%$, although collectively these three cancers kill fewer people each year than lung cancer. ${ }^{12}$ The disproportionately low funding for lung cancer extends into other areas of respiratory medicine and this has been recognised as a problem by the Medical Research Council (www.mrc.ac.uk).

In summary, the second edition of "The Burden of Lung Disease" is a timely reminder of the needs of people with respiratory disease in the UK. The report highlights areas where more evidence is required. This means that researchers working in respiratory medicine need to maximise their ability to secure the limited research funding available by working together to produce high quality proposals to answer clinically relevant questions. In many areas we already have enough clinical evidence to help reduce the mortality and morbidity of patients with lung disease, but the current service provision is fragmented. Here we need a coordinated effort from the whole of the respiratory community to push for the services our patients deserve.

Thorax 2006;61:557-558.

doi: $10.1136 /$ thx. 2006.066050

Correspondence to: Professor R Hubbard, British Lung Foundation Professor of Respiratory Epidemiology, Division of Epidemiology and Public Health, University of Nottingham, Nottingham NG5 1PB, UK; richard.hubbard@ nottingham.ac.uk

\section{REFERENCES}

1 British Thoracic Society. The burden of lung disease: a statistical report from the British Thoracic Society, 2nd ed. London: British Thoracic Society, 2006.

2 Stradling JR, Crosby JH. Predictors and prevalence of obstructive sleep apnoea and snoring in 1001 middle aged men. Thorax 1991:46:85-90.

3 Yaggi HK, Concato J, Kernan WN, et al. Obstructive sleep apnea as a risk factor for stroke and death. N Engl J Med 2005;353:2034-41.

4 Stradling JR, Davies RJ. Sleep-1: Obstructive sleep apnoea/hypopnoea syndrome: definitions, epidemiology, and natural history. Thorax 2004;59:73-8.

5 Johnston I, Britton J, Kinnear W, et al. Rising mortality from cryptogenic fibrosing alveolitis. BMJ 1990;301:1017-21.

6 Hubbard R, Johnston I, Coultas DB, et al. Mortality rates from cryptogenic fibrosing alveolitis in seven countries. Thorax 1996:51:711-6.

7 Royal College of Physicians. Nicotine addition in Britain. A report of the Tobacco Advisory Group of the Royal College of Physicians. London: Royal College of Physicians, 2000.

8 Gordon P, Sanders MH. Sleep-7: Positive airway pressure therapy for obstructive sleep apnoea/hypopnoea syndrome. Thorax 2005;60:68-75.

9 Scott S, Walker P, Calverley PM. COPD exacerbations -4 : Prevention. Thorax 2006;61:440-7

10 Imperatori A, Harrison RN, Leitch DN, et al. Lung cancer in Teesside (UK) and Varese (ltaly): a comparison of management and survival. Thorax 2006;61:232-9.

11 Gleeson FV. Is screening for lung cancer using low dose spiral CT scanning worthwhile? Thorax 2006:61:5-7.

12 Partners in Cancer Research. Strategic analysis 2002: an overview of cancer research in the UK directly funded by NCRI partner organisations. London: NCRI, 2002 